\title{
Excessive Dynamic Airway Collapse and Tracheobronchomalacia Increase the Frequency of Hospitalization in COPD Patients
}

In this issue, you are going to read an article written by Yarkın et al. (1) that discusses excessive dynamic airway collapse as a cause for frequent admission to the hospital in chronic obstructive pulmonary disease (COPD). The authors state that it is controversial whether excessive dynamic airway collapse seen in COPD is a complication of the disease or a comorbidity accompanying COPD but think that the collapse plays an important role in the progression and the course of COPD. This study of the authors, which investigated whether or not there is a relationship between the admission frequency of patients who are admitted more than twice in the same year and the existence of dynamic airway collapse, deserves to be mentioned and discussed, despite its certain constraints, because it emphasizes an important point. In this short editorial, the relationship between excessive dynamic airway collapse and tracheobronchomalacia (TBM) should especially be kept in mind among the conditions accompanying COPD, and possible treatment options will be summarized.

Tracheobronchomalacia is a disease of the main airways. It is caused by the weakening of the tracheal or bronchial wall due to softening or damaging of the cartilage-supporting tissue and/or degeneration or atrophy of the elastic fibers in the outer layer. The trachea and bronchia, therefore, collapse excessively in expiration, and the lateral circumference of the airway decreases by at least $50 \%$ (2). Another term, excessive dynamic airway collapse, is described as pronounced constriction (constricting the lumen by more than $50 \%$ ) compared to what should normally be observed in expiration. The main pathology here is thought to be in the posterior membrane. However, authorities emphasize that practically, both clinical cases cause constriction in a similar way and that the treatment approach does not differ (3). The main symptoms in both cases are dyspnea, orthopnea, chronic cough, and difficulty in producing sputum. In both cases, the main result is prominent constriction in the airway lumen in expiration, and the treatment approaches are similar.

In tracheomalacia, there is dynamic obstruction in the airways; therefore, in pulmonary parenchyma, hyperinflation and air trapping may result to a certain degree. In normal expiration, increased pressure in the thoracic cage causes constriction in the airways, but this constriction is balanced by the rigidity of the trachea and bronchial tree, the intraluminal pressure, and the cartilage-supporting tissue. The intrathoracic pressure that keeps rising in tracheobronchomalacia exceeds the intraluminal pressure and, by reducing airflow, causes dyspnea, difficulty in producing sputum, recurring infections, and possible chronic respiratory failure (3). It is controversial whether COPD causes dynamic constriction or whether the existence of dynamic constriction should be considered a factor that exacerbates COPD.

Some authors judge the dynamic obstruction observed in chronic obstructive pulmonary disease as the dissemination of a peripheral obstruction. Differing degrees of central airway malacia are observed in a substantial part of cases with severe emphysema (3).

In their study, which involves 71 COPD cases and makes use of multidetector computed tomography (MDCT), Sverzelatti et al. (4) detected malacia in 38 cases (53\%). In this study, a relationship was not found between the existence of emphysema and bronchiectasis and malacia. However, it was determined that the increase of bronchial wall thickness correlated with malacia and that the collapse increased as thickness increased. On the other hand, in their study, again making use of multidetector computed tomography (MDCT), O’Donnell et al. (5) determined expiratory collapse on average $17 \%$ of the time after expiration and $62 \%$ of the time during forced expiration. Inoue et al. (6) studied the existence of tracheobronchomalacia in emphysema cases, again with MDCT taken during inspiration and expiration, and determined that in 4 of the 56 consecutive patients, the airway lumen was obstructed more than $50 \%$ of the time after expiration. They found a tracheobronchomalacia frequency among the case population of $7.1 \%$. In light of these studies, it can be said that that there is an association that is not rare between COPD and the existence of tracheobronchomalacia and dynamic excessive collapse. In another interesting study, Boiselle et al. (7) studied obese cases and compared them with a healthy control group. In this study, the existence of tracheal collapse in obese cases with COPD was statistically significantly higher than in non-obese COPD cases. However, the same difference was not detected in healthy patients who were and were not obese. These findings lead one to think that obesity accompanying COPD increases the dynamic obstruction of the airway. 
Loring et al. (8) tried to determine the relationship between the the collaps of the main airways and its contribution to the reduction of airflow in patients with asthma and COPD. In this study, of the 80 patients with suspected or confirmed TBM, it was determined that $40 \%$ had COPD and $24 \%$ had asthma. No correlation was found between the degree of obstruction, measured by gas volume exhaled at the first second of expiration ( $\left.\mathrm{FEV}_{1}\right)$, and main airway collapse. In other similar studies, tracheal collapse was found to have only limited contribution to the total resistance of the airways $(3,8)$. Recent studies suggest that COPD, too, is recovered in patients who undergo serious TBM treatment (9).

Screening of dynamic expiratory collapse is the golden standard in the diagnosis of $\operatorname{TBM}(2,3)$. The preferred method is flexible bronchoscopy, either under local anesthesia or after mild intravenous sedation. Meanwhile, the patient can perform spontaneous respiration and follow instructions; therefore, the responses of the trachea during spontaneous respiration can be determined (2).

In their study, Yarkın et al. studied 34 cases and found the average annual admission rate to be 3.3 and defined those who were admitted twice a year and those who were admitted more than twice as two different groups and compared them. Bronchoscopy was performed in all cases, and excessive dynamic collapse was determined in 17 cases (50\%). When the two groups were compared in this regard, the existence of collapse in the group that was admitted more than twice a year was $72 \%$ and $8 \%$ in the other group (just 1 case). In the regression analysis, it was statistically shown that the existence of excessive dynamic collapse is an independent variable that increases the frequency of admission. The authors do not recommend anything for the treatment of these cases. Given that the main emphasis of the study was the existence of excessive dynamic collapse as an additional exacerbating factor that worsens the picture in COPD, this discussion can be accepted as the topic of another study.

Ernst et al. (10) prospectively observed 75 patients that were treated with a silicon $Y$ stent due to diffuse and severe TBM. They evaluated the subjective symptoms prior to and 14 days after placing the stent, the quality of life, and the tolerance to exercise with the 6-minute walk test. In some cases, the stent resulted in definitive treatment, but in some cases, it helped identify the patients that could be helped with the application of tracheobronchoplasty. After the first month, there were significant improvements in symptoms and quality of life, but no improvement was observed in the parameters of the pulmonary function test or the 6-minute walk test. Also, the numerousness of the complications caused by the stents was observed, and they had to be removed in most of these complications.

In another study, Murgu et al. (11) found that despite the initial improvement in symptomology and pulmonary functions, the level of complications was very high in 15 patients with severe TBM that was treated with silicon stents.

Stents should only be used in patients with severe symptomologies and ones that can be followed up with frequent bronchoscopies in order to predict every kind of complication and to establish their treatment $(2,10,11)$. In patients with severe symptoms that are candidates for surgical treatment, the stent is used as a treatment test that identifies the benefits of fixing the airway circumference.
On the other hand, if symptomatic recovery is established, stents can be used as a long-term palliative treatment for patients that have no alternative treatments, provided that follow-up and care of the stent are performed.

In our unpublished series, consisting of 8 cases where we applied stent treatment, only 2 cases benefited from the stent, and frequent admissions to the intensive care were eliminated, but intensive mucositis in the remaining 6 cases rendered the management of the stent impossible, and the stents had to be removed.

The endoscopic treatment of tracheobronchomalacia and the silicon stent application were extensively studied in previous years. Even though these methods initially offered some improvements in symptoms and quality of life, very minimal improvements could be had in pulmonary function, and there are no data regarding the long-term benefits. However, given that the complication rate is very high, it is clear that stents can not be the reference treatment in these diseases (12). Stents, just like in all of its indications, can have a palliative effect in the absence of etiological medical treatment and radical surgical treatment. New endoscopic treatments, such as laser, offer intriguing perspectives in the stabilization of the tracheobronchial membrane. In the future, it is possible that active and/or biodegradable stents that are currently in the research and review phases will find an application area in TBM.

In conclusion, in COPD cases with frequent admissions, the existence of excessive dynamic airway collapse, and tracheobronchomalacia should be kept in mind, and the diagnosis should be confirmed both by dynamic MDCT studies taken in expiration and inspiration and by bronchoscopy. In these cases, there is no consensus about the treatment that should be performed in addition to COPD treatment, but it should not be forgotten that in select cases, surgical options or application of a stent can be a solution.

\section{Levent Dalar}

Department of Chest Diseases, İstanbul Bilim University Faculty of Medicine, İstanbul

Address for correspondence: Levent Dalar, Department of Chest Diseases, İstanbul Bilim University Faculty of Medicine, İstanbul, Turkey E-mail: leventdalar@yahoo.com

Received date: 27.09 .2014

Accepted date: 28.09.2014

Available Online Date: 02.12.2014

C Copyright 2014 Turkish Respiratory Society (TRS)

DOI: 10.5152/ejp.2014.18209

- Available online at www.eurasianjpulmonol.com

\section{REFERENCES}

1. Yarkın T, Ağca M, Acar G, Göl G, Tokgöz F, Baran R. Investigation of the relationship between the frequency of hospitalization and comorbidity and excessive dynamic airway collapse in frequently hospitalized COPD patients. Eurasian J Pulmonol 2014; 16: 169-74.

2. Dutau H, Dalar L. Erişikinlerde trakebronkomalazi. In:Trakea ed: Bedirhan MA. Probiz Ltd. Şti. İstanbul 2012; 55-72.

3. Majid A, Fernández L, Fernández-Bussy S, Herth F, Ernst A. Tracheobronchomalacia. Arch Bronconeumol 2010; 46: 196-202. 
4. Sverzellati N, Rastelli A, Chetta A, Schembri V, Fasano L, et al. Airway malacia in chronic obstructive pulmonary disease: prevalence, morphology and relationship with emphysema, bronchiectasis and bronchial wall thickening. Eur Radiol 2009; 19: 1669-78.

5. O'Donnell CR, Bankier AA, O'Donnell DH, Loring SH, Boiselle PM. Static end-expiratory and dynamic forced expiratory tracheal collapse in COPD. Clin Radiol 2014; 69: 357-62.

6. Inoue M, Hasegawa I, Nakano K, Yamaguchi K, Kuribayashi S. Incidence of tracheobronchomalacia associated with pulmonary emphysema: detection with paired inspiratory-expiratory multidetector computed tomography using a low-dose technique. Jpn J Radiol 2009; 27: 303-8.

7. Boiselle PM, Litmanovich DE, Michaud G, Roberts DH, Loring SH, et al. Dynamic expiratory tracheal collapse in morbidly obese COPD patients. COPD 2013; 10: 604-10.
8. Loring SH, O Donnell CR, Feller-Kopman DJ, Ernst A. Central airway mechanics and flow limitation in acquired tracheobronchomalacia. Chest 2007; 131: 1118-24.

9. Kandaswamy C, Balasubramanian V. Review of adult tracheomalacia and its relationship with chronic obstructive pulmonary disease. Curr Opin Pulm Med 2009; 15: 113-9.

10. Ernst A, Majid A, Feller-Kopman D, Guerrero J, Boiselle P, Loring S, etal. Airway stabilization with silicon stents for treating adult tracheobronchomalacia: A prospective observational study. Chest 2007; 132: 609-16.

11. Murgu SD, Colt HG. Complications of silicone stent insertion in patients with expiratory central airway collapse. Ann Thorac Surg 2007; 84: 1870-7.

12. Freitag L. Airway Stents. In: Strausz J, Bolliger CT. Interventional Pulmonology. Eur Respir Mon 2010, 18: 190-217. 